\title{
Poliartritis asociada a leishmaniasis en un canino del nordeste argentino
}

\author{
Lockett, M.B.'; Llano, E.G. ${ }^{2}$; Maidana, H.R. 3; Báez, A.D. ${ }^{3}$; Cabrera, W.R. ${ }^{2}$ \\ ${ }^{1}$ Hospital de Clínicas, ${ }^{2}$ Cátedra Anatomía II y ${ }^{3}$ Clínica de Pequeños Animales, \\ Fac. Cs. Veterinarias, UNNE, Cabral 2139, Corrientes (3400), Argentina. \\ E-mail: marielveter@hotmail.com
}

\begin{abstract}
Resumen
Lockett, M.B.; Llano, E.G.; Maidana, H.R.; Báez, A.D.; Cabrera, W.R.: Poliartritis asociada a leishmaniasis en un canino del nordeste argentino. Rev. Vet. 30: 1, 28-31, 2019. En una de las etapas de la leishmaniasis se produce una reacción inmunomediada, con formación de complejos inmunes que se depositan -entre otros lugares- a nivel articular. El objetivo del presente trabajo fue reportar los hallazgos radiológicos observados en un canino hembra, mestizo, con diagnóstico positivo de leishmaniasis y signos de enfermedad articular, entre otros propios de la infección. Radiológicamente se observaron alteraciones erosivas en las articulaciones de ambos lados del carpo y del tarso. El nordeste argentino es considerado como un área de transmisión moderada a intensa de leishmaniasis, razón por la cual los infrecuentes signos de presentación de patologías osteoarticulares deben ser tenidos en cuenta en esta enfermedad. La importancia de las manifestaciones radiológicas reside en el hecho de que reflejan la anatomía patológica macroscópica de la enfermedad y permiten identificar la etapa en la que se encuentra la lesión a nivel articular, posibilitando la realización un diagnóstico diferencial con otras artropatías.
\end{abstract}

Palabras clave: perro, articulación, análisis clínicos, leishmaniasis, radiología.

\begin{abstract}
Lockett, M.B.; Llano, E.G.; Maidana, H.R.; Báez, A.D.; Cabrera, W.R.: Polyarthritis associated to leishmaniasis in a canine of northeastern Argentina. Rev. Vet. 30: 1, 28-31, 2019. In one of the stages of leishmaniasis an immune-mediated reaction occurs, with formation of immune complexes that are deposited at the joint level, among other places. The objective of the present work was to report the radiological findings observed in a canine, female, mixed, with a positive diagnosis of leishmaniasis and signs of joint disease. Radiologically, erosive alterations were observed in the joints of both sides of the carpus and tarsus. The northeastern region of Argentina is considered as a moderate to intense transmission area of leishmaniasis, the infrequent presentation signs of osteoarticular disease must be taken into account. The importance of these radiological manifestations relies in the fact that they reflect the macroscopic pathological anatomy of the disease and allows to identify the stage in which the lesion is located at the articular level, making it possible to carry out a differential diagnosis with other arthropathies.
\end{abstract}

Key words: dog, joint, clinical analysis, leishmaniasis, radiology.

\section{INTRODUCCIÓN}

El elemento patogénico primario en la leishmaniasis canina es la infección y multiplicación del parásito en el sistema fagocítico mononuclear, donde se disemina por vía hemática o linfática a todo el organismo. Existe una segunda instancia en el curso de la enfermedad en la cual se produce una reacción inmunomediada, que ocasiona la formación de complejos inmunes.

Dichos complejos se depositan en lugares como riñones, pulmones, plexo coroides del sistema nervioso

Recibido: 15 mayo 2018 / Aceptado: 12 setiembre 2018 central, procesos ciliares del ojo y articulaciones, entre otros. Ello provoca una reacción de hipersensibilidad tipo III que implica a las estructuras comprometidas, las que desarrollan alteraciones estructurales y funcionales ${ }^{3,5}$.

En los pequeños animales, la distribución poliarticular es una característica de las artropatías inflamatorias con base inmune. La mayoría de las restantes enfermedades articulares afectan una o pocas articulaciones ${ }^{6}$.

Solo el $30 \%$ de los animales afectados por leishmaniasis desarrollan problemas locomotores. Claudicación, artralgia, edema, rigidez articular, paresia, atrofia 
muscular e intolerancia al ejercicio, son algunos de los síntomas que se pueden manifestar cuando se comprometen las extremidades por esta enfermedad ${ }^{3,4}$.

En las artropatías de caninos con leishmaniasis los signos radiográficos varían a lo largo del tiempo. Inicialmente puede existir efusión sinovial, distensión de la cápsula y aumento de los espacios articulares. Más tarde, en los procesos erosivos, se produce la destrucción del cartílago y la reducción del tamaño de los espacios articulares. Reacciones periósticas, inflamación de los tejidos blandos periarticulares y cambios de densidad ósea son característicos de los estadios avanzados. Las articulaciones de las extremidades son afectadas en forma bilateral y simétrica ${ }^{1}$.

El objetivo de la presente comunicación fue reportar los hallazgos radiológicos observados en las articulaciones de un canino con leishmaniasis en la región del nordeste argentino.

\section{MATERIAL Y MÉTODOS}

En el Hospital de Clínicas de la Facultad de Ciencias Veterinarias de Corrientes (Argentina), se presentó a la consulta un canino de raza indefinida, de un año de edad, con escoriaciones en la punta de las orejas, conjuntivitis, debilidad, caquexia, onicogrifosis, cojera (más manifiesta en miembros posteriores) y respuestas dolorosas al examen de las articulaciones de carpo y tarso (Figura 1).

Se tomaron muestras de sangre de la vena cefálica antibraquial para determinar los valores del hemograma y detectar eventuales modificaciones bioquímicas de los parámetros séricos. También se investigó la presencia de amastigotes en el líquido sinovial de las articulaciones comprometidas, así como en el extremo distal de la médula ósea de la última costilla, para realizar pruebas ante la sospecha de leishmaniasis.

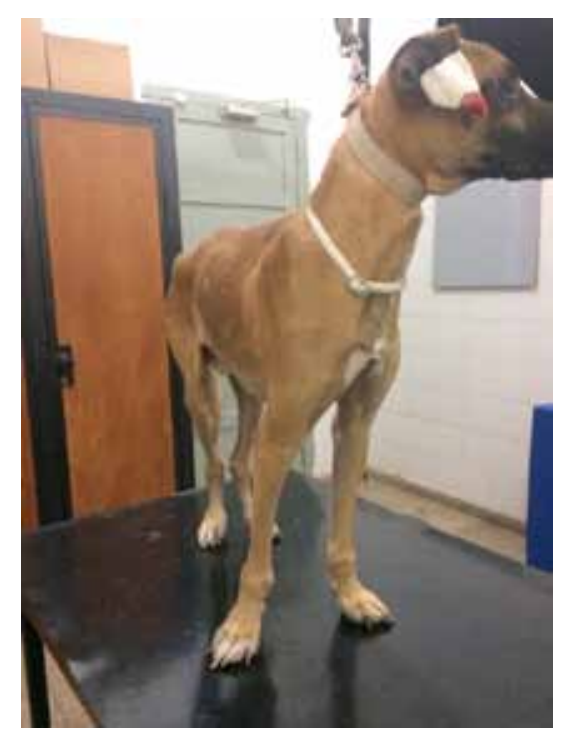

Figura 1. Aspecto general del paciente. Nótese el mal estado de nutrición, la atrofia muscular y la hinchazón articular de las extremidades
Para la detección de anticuerpos contra Leishmania $s p$ se utilizó un método serológico cualitativo indirecto (test rápido inmuno-cromatográfico Kalazar Detect Canine-rK 39). La evaluación radiográfica se realizó en las articulaciones de carpo y tarso. Se usaron chasis de un tamaño de $24 \times 30 \mathrm{~cm}$ con pantalla de fósforo y una unidad radiológica portátil de alta frecuencia 100 TP. 100 mA/110 Kvp. Para el procesamiento de las películas se utilizó un procesador digital.

\section{RESULTADOS}

Los resultados de laboratorio no revelaron grandes anormalidades en los parámetros hematológicos y bioquímicos. El recuento de eritrocitos fue de 5.870.000/ $\mathrm{mm}^{3}$, el de leucocitos resultó de $16.700 / \mathrm{mm} 3$, la concentración de hemoglobina arrojó $10,6 \mathrm{~g} / \mathrm{dl}$, el hematocrito fue de $35 \%$, proteinemia y uremia revelaron $7,3 \mathrm{~g} /$ dl y $0,35 \mathrm{~g} / 1$ respectivamente, y las actividades enzimáticas de GPT, GOT y fosfatasa alcalina fueron de 16,3; 11,4 y $543 \mathrm{U} / \mathrm{L}$.

La evaluación del líquido sinovial mostró un aumento de la celularidad. Las células detectadas fueron neutrófilos $(60 \%)$, células mononucleares $(16 \%)$ y linfocitos (8\%). Tanto en macrófagos como en neutrófilos, se observaron amastigotes de Leishmania sp en el citoplasma.

El canino dio reactivo ante el método indirecto y se confirmó el diagnóstico en el método directo, donde se detectó la presencia del amastigotes en la muestra de medula ósea.

Radiológicamente se diagnosticó poliartritis erosiva multifocal de predominio carpo-tarsal bilateral. En la articulación del carpo se observó erosión ósea pericondral, pérdida de mineralización e irregularidad de los bordes articulares, así como aumento del volumen y densidad del tejido blando de la zona, con reducción del tamaño de los huesos del carpo (Figura 3).

En el tarso se visualizó pérdida de densidad del hueso subcondral, proliferación perióstica periarticular y disminución de la densidad de los componentes óseos de la articulación, los cuales revelaron un trabeculado grosero (Figura 4).

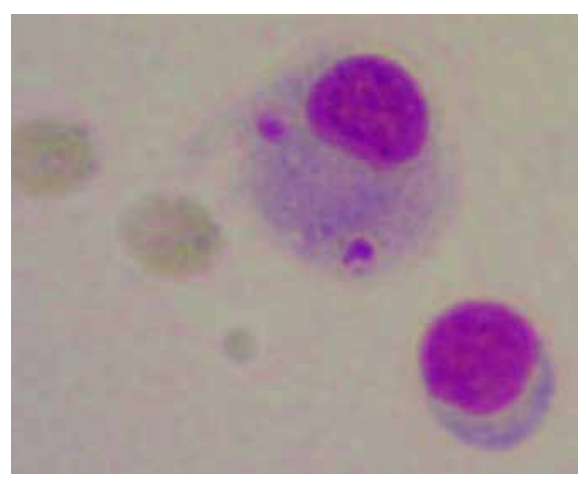

Figura 2. Análisis parasitológico de líquido sinovial con identificación de macrófagos conteniendo amastigotes de Leishmania sp. Obj. 100x. 


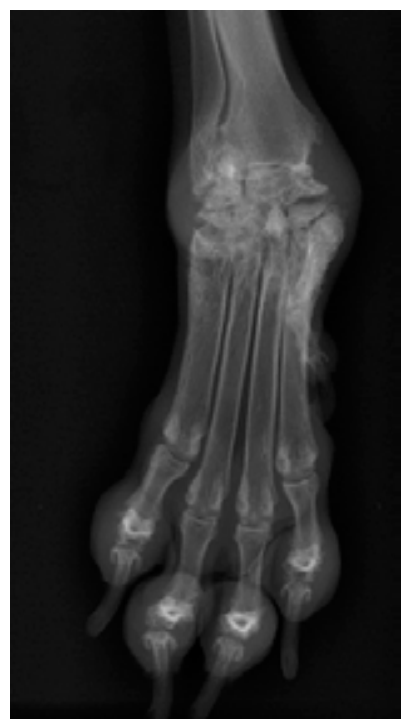

Figura 3. Radiografía de la articulación del carpo con incidencia anteroposterior. Se observa esclerosis subcondral, trabeculado grosero, alteración de la forma y tamaño de los huesos carpianos.

El paciente fue tratado con analgésicos, allopurinol (10 mg/kg/24 h) y domperidona ( $0,5 \mathrm{mg} / \mathrm{kg} / 24 \mathrm{~h})$. Durante los 6 meses siguientes se registró una mejoría del estado general del animal, tras lo cual empezó a decaer y el propietario decidió sacrificar al animal.

\section{DISCUSIÓN}

La presencia de un infiltrado inflamatorio articular compuesto por macrófagos parasitados por Leishmania $s p$, postula que esta invasión parasitaria fue el origen de la enfermedad articular.

La leishmaniasis puede provocar lesiones articulares en los caninos, que aparecen como manifestación de una reacción de hipersensibilidad tipo III, en la cual las enzimas liberadas por las células inflamatorias, sinoviocitos y condrocitos, dañan al cartílago articular fomentando la aparición de anormalidades erosivas ${ }^{2,11}$.

Una de las características de estas artropatías es que las alteraciones radiológicas se aprecian simultáneamente en las áreas articulares homólogas de ambas partes del cuerpo. Así ocurrió en el presente caso, donde las articulaciones del carpo y tarso fueron afectadas bilateralmente.

Los hallazgos detectados en las imágenes radiográficas fueron similares a los reportados por otros investigadores, incluyendo la presencia de reacción perióstica, destrucción del cartílago articular, compromiso del hueso subcondral, inflamación de los tejidos blandos periarticulares y cambios de densidad de los componentes óseos de las articulaciones comprometidas.

El nordeste argentino es considerado actualmente como un área de transmisión moderada a intensa de leishmaniosis visceral canina. La variedad de síntomas y las diferentes manifestaciones que presenta esta enfermedad tornan dificultoso el diagnóstico clínico, pudiendo confundirse con otras patologías. En los perros

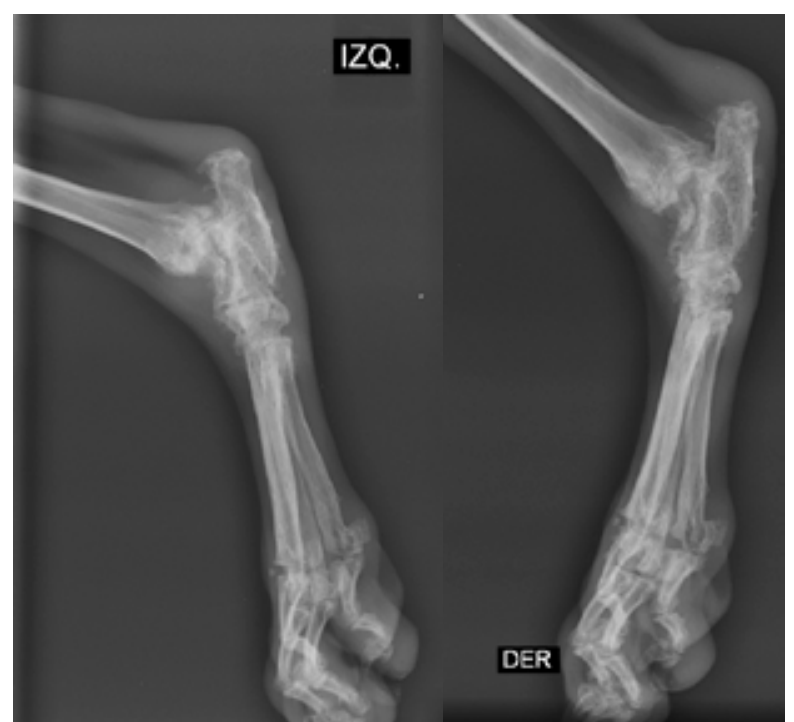

Figura 4. Radiografia latero-lateral de las articulaciones tarsales izquierda y derecha. Se evidencian zonas de osteólisis, colapso articular y reacción perióstica.

se puede presentar como una infección subclínica, una enfermedad autolimitada o una afección grave no autolimitada. El parasitismo tiende a ser más intenso en los animales que manifiestan una presentación clínica más severa.

Existen dudas con respecto a que la presentación inusual de estos signos osteoarticulares guarde relación con la intensidad de la infección (masiva o de baja intensidad) y con la duración del proceso. En el presente caso, si bien no se pudo determinar con certeza el tiempo de evolución de la enfermedad, se consideró que era avanzada dada la magnitud de las alteraciones constatadas ${ }^{7,10}$.

En un estudio realizado sobre 14 pacientes positivos a Leishmania $s p$ con artritis inflamatoria asociada, el $36 \%$ de los casos cursó con monoartritits y el $28 \%$ con poliartritis, siendo el carpo la articulación más comprometida. Ello difiere con los hallazgos del presente caso, donde los tarsos fueron los más afectados.

En una investigación similar, solamente la mitad de los caninos estudiados presentó signos radiográficos evidentes, debido a que la manifestación radiológica de la poliartritis ocurre en pacientes crónicos. Igualmente, el proceso de la enfermedad es variable en cada individuo, pudiendo evolucionar de forma diferente a pesar de presentar la misma etiología, debido a factores genéticos, diferencias en el sistema inmunitario o respuestas inapropiadas frente a diversos antígenos ${ }^{8,9}$.

La importancia de estas manifestaciones radiológicas reside en el hecho de reflejar la anatomía patológica macroscópica de la enfermedad y permitir identificar la etapa en la que se encuentra la lesión a nivel articular, posibilitando la realización de un diagnóstico diferencial con otras artropatías.

La presencia de una dificultad en la locomoción en un paciente positivo a Leishmania $s p$ tendría que inducir la sospecha de una enfermedad inflamatoria de 
la articulación. La cojera puede no estar presente en todos los casos de pacientes con artritis asociada con leishmaniasis, por lo cual estaría indicado realizar un examen ortopédico cuidadoso y un análisis de líquido sinovial.

Futuros trabajos con un mayor número de pacientes deberán realizarse para determinar el valor estadístico de los hallazgos en la zona y definir si ellos pueden modificar la conducta clínica y terapéutica en los animales afectados.

Agradecimientos. Por su colaboración y aporte en el manejo del paciente, al alumno Eduardo Ariel Mouchard.

\section{REFERENCIAS}

1. Barreiro A, Villa M. 2014. Interpretación radiológica del sistema óseo. En: Diagnóstico por imagen en pequeños animales (Agut Jiménez A. Ed.), Multimédica Ediciones Veterinarias, Barcelona, p. 109-161.

2. Cooper BJ, Valentine BA. 2016. Muscle and tendon. In: Pathology of domestic animals (Palmer, Jubb \& Kennedy), $6^{\text {th }}$ ed., Elsevier, USA, vol. 1, p. 240.

3. Dennis R, Kirberger RM, Wrigley RH, Barr FJ. 2010. Handbook of small animal radiology and ultrasound, $2^{\text {nd }}$ ed. (eBook ISBN 9780702043970), Saunders-Elsevier, Edinburgh, p. 39-42.
4. Graeme A, Nicoll R. 2006. Distal limbs carpus and tarsus. In: BSAVA Manual of canine and feline musculoskeletal imaging (Barr FJ, Kirberger RM), $1^{\text {st }}$ ed., Wiley, London, p. $150-167$.

5. Lappin MR. 2005. Protozoal and miscellaneous. In: Textbook of veterinary internal medicine (Ettinger SJ, Feldman EC), $6^{\text {th }}$ ed., Elsevier, Missouri, USA, p. 644-645.

6. Nelson RW, Couto CG. 2010. Medicina interna de pequeños animales, $4^{\circ}$ ed., Elsevier, Barcelona, p.1127-1140.

7. Santos RL, Costa EA, Pangrazio KK. 2010. Genital lesions and venereal transmission of canine visceral leishmaniasis. In: Veterinary Parasitology (LaMann GV), Nova Science Publishers, New York, p. 193-200.

8. Sbrana S, Marchetti V, Mancianti F, Guidi G, Bennetti D. 2014. Retrospective study of 14 cases of canine arthritis secondary to leishmania infection. J Small Anim Pract 55: 309-313.

9. Soares AR. 2009. Avaliação radiográfica das articulações dos membros locomotores de cães naturalmente acometidos por leishmaniose visceral no município de Araçatuba-SP. Dissertação (mestrado), Universidade Estadual Paulista, São Paulo, p. 25-38.

10. Solano GL et al. 2009. Directions for the diagnosis, clinical staging, treatment and prevention of canine leishmaniosis. Vet Parasitol 165: 1-18.

11. Turrel JM, Pool R. 1982. Bone lesions in four dogs with visceral leishmaniasis. Vet Radiol 23: 243-249.

\section{Revista Veterinaria obtuvo el máximo nivel de categorización del CAICYT-CONICET}

Tras el pertinente proceso de evaluación según criterios de calidad editorial, en setiembre de 2005 CAICYT-CONICET ha clasificado a nuestra publicación con Categoría 1 (nivel superior de excelencia), con lo cual pasa a integrar el Catálogo Latindex (folio 14022). La Dirección de Revista veterinaria agradece a quienes colaboraron para obtener tan importante distinción. Ver: http://www.latindex. unam.mx/busquedas/catalogotitulo.html 